\title{
Research on Application of Mobile Context-Awareness in Recommendation System
}

\author{
Jie Ma \\ Hainan College of Software Technology, Hainan, Qionghai, 571400
}

\begin{abstract}
Keywords: Mobile Context-Awareness, Recommendation System, Application Status
\end{abstract}
\begin{abstract}
With the advent of the communications era, mobile devices and other mobile devices becomes the main tool for communication and data access between people. But mobile phones and other mobile devices still exist complicated operation, the service object is not accurate and a series of shortcomings. Context-aware theory is through the sensor and its related technology so that computers and other equipment can "perceive" to the user in a variety of situations. In this paper, through the study of these situational information, to further perceive the user's behavioral motivation, etc., from which users find the law of the product, and these laws and product development research combined, and then found that the current product development problems may exist, recommended for mobile terminals The design of the system is developed according to the design specification.
\end{abstract}

\section{Introduction}

The computer can not understand the human language, can not understand how the human world works and can not perceive the current form information. In order to allow the computer to work in accordance with the needs of the human body, the computer needs to be translated, clear what to do the details and practices, mainly through the keyboard and mouse to set the details for the computer. Can we improve the computer so that we can get the situation, further perceive the situation, enrich the human-computer interaction to provide better computing services? The answer is yes. This is Context Awareness and contextual sensing. With the popularity of Internet of things sensors and cloud computing technology gradually mature, sensor-aware technology and massive data processing problems are no longer insurmountable. After collecting the analysis of sensors and other sources of information, the user's circumstances will be able to be computer identification, and even a certain period of time historical records will be perceived, to provide users with "think of the" information and services.

\section{The Concept of Context Awareness}

For context, what is currently commonly cited is DEY [25] in its doctoral thesis on the definition of Context: context is used to characterize a physical state of any information. The object can be a person, a location, or an object that is considered to be related to the interaction between the user and the application, including the user and the application itself. Emphasis on the situation actually reflects the shift from a computer-centric to a people-centered approach. Therefore, it should be people-oriented, around the user (person) to consider the situation.

Reference dey to the definition of context awareness: whether it is a desktop computer or mobile devices, pervasive computing environment in the use of context applications, are called context awareness. He defines the context-aware system as: the use of context to provide relevant information or services to users, where the associated things depend on the user's task, which is Context Awareness. Context perception can be divided into active Context Awareness and passive context perception. In fact, the context perception is divided into direct explicit perception and internal implication perception, such as location information, time information and equipment environment information, the latter, such as user characteristics, habits, knowledge level and preferences. Among them, the three context awareness behavior is very important, namely: the user information and service representation, the automatic implementation of the service, marking the 
situation for later retrieval.

Context-aware computing is the use of environmental features such as the user's location, time, logo and activities, inform the computing device, so that users can provide information related to the current situation. Pervasive computing is closely related to context-dependent computing, but it can not be said that context-aware computing is pervasive computing. Context-aware computing is more general than pervasive computing, such as predicting what users want to do more in order to give users more direct help. Such systems are also common on conventional desktop systems, which are not in the scope of pervasive computing within. In the final analysis, Context Awareness computing is a new form of computation that is closely related to pervasive computing, mobile computing, and intelligent computing.

\section{The Key Technology of Mobile Terminal Application based on Context Awareness}

In the environment to perceive the user's context, to provide personalized information and services, an important prerequisite is to have context-aware data mining capabilities. From the location, time, merchandise, the surrounding environment, user characteristics and other aspects of the user in the context or the next context of the individual needs, so as to provide targeted services. From the domestic and international research status, Context Awareness research is still in the initial stage, and specifically for the context of the business of data mining research is very few. However, the rapid development of mobile Internet and user needs makes the research of context-aware data mining become more and more important.

In recent years, data mining has attracted the attention of the information industry, mainly because the massive data available in the Internet can be widely used, and the urgent need to convert these data into useful information and knowledge. Data-rich, accompanied by a need for powerful data analysis tools, is described as "data-rich, but information-poor." The rapid growth of massive data collection, stored in large and large databases, does not have powerful tools to understand that they have gone far beyond human capacity. Data mining tools for data analysis and can be found in important data model, access to information and knowledge can be applied to all walks of life, including business management, production control, market analysis, engineering design and scientific exploration. Data mining is a non-trivial process of obtaining effective, novel, potentially useful, and ultimately understandable patterns from large amounts of data stored in databases, data warehouses, or other repositories. In the field of artificial intelligence, it is also known as the Knowledge Discovery Database (KDD) in the database, and some people regard data mining as a basic step in the knowledge discovery process in the database. The knowledge discovery process consists of three phases: data preparation, data mining, results representation and interpretation. Data mining can interact with users or knowledge bases.

The measurement of network behavior is the observation and evaluation of network behavior, the information obtained by the measurement technology, and it is important to understand some unknown behavior of this complex environment composed of known technologies. Through the network user behavior measurement and analysis, to understand the network operating environment applications and services to the actual working conditions, to find specific characteristics of the user or user groups. The measurement of network traffic is also an important means to study the behavior of network users. At present, domestic and foreign related literature on the network traffic measurement conducted in-depth and meticulous research. Some of them describe the concept of network measurement, pointing out that the direct purpose of network measurement is to obtain traffic data related to network operation. The measurement of these traffic data is also the basis for network user behavior measurement. How to carry on the online real-time measurement to the behavior of the network user is the key problem that the online network user behavior analysis needs to solve.

According to the statistics of network traffic and association rules analysis, you can find the user's Internet habits, for example, subway stations, bus stations and airports are mobile Internet users more concentrated place, that is, the same time the network traffic value relative to other places larger. At this point, businesses can use this advantage to promote their products and develop 
markets, such as SMS advertising and so on. When the number of potential customers becomes larger, it is undeniable that companies have the opportunity to win the initiative. In addition, operators can use the network traffic data to find the frequent itemsets of user operations, that is, the relationship between the user's relationship shows the universality of user behavior, in order to determine the same background, what products and information is Most users are in desperate need. For example, passengers at buses such as bus stops may receive short messages about the bus from their mobile phones so that they can understand the current traffic situation, how many minutes his car will arrive, and so on. For example, in the modern office building, as more and more electrical equipment is purchased, the building electricity consumption is also rising month by month. When most of the staff members get off work, a few people remain in the room to work overtime. , The use of a wide range of lights and air conditioning will produce unnecessary power consumption and therefore, to reduce the overall building energy consumption is an urgent need to be addressed. The emergence of conventional technology products, such as the appearance of voice lights, has reduced the consumption to a certain extent, but this is not enough, and the contextual perception through mobile terminals may be able to better solve this problem. Imagine, when the user carries the mobile terminal to carry the information and analysis of the surrounding environment, these operations will produce a certain amount of network traffic, the building's internal server according to the relevant statistics issued instructions, command the building within the relevant power system automatically response. For example, if the staff in a conference room only meet in the meeting room, then their mobile phone to perceive their location, after the summary, the server issued a directive to other room lights closed, heating or directly close the other room Air conditioning to save unnecessary waste of electricity.

The limitations of the equipment various types of mobile phone equipment input and interaction interface are not the same, limited screen space, difficult text input method or even continuous network coverage will be the application of the popularity of the product hinder, today's Mobile users are not too much time and patience to face these difficulties. Network gap still exists Although the network transmission speed gradually increased, but the huge flaw is still there, mobile terminal users want to get more information at a time, sometimes even in the network can be outside the scope of coverage, and It is undeniable that large-scale synchronous data transmission is always slow and inadequate. The user's choice proves that mobile users are reluctant to pay more money to buy the application. Most of the users in China prefer free applications from businesses. The practice is that operators provide free services to users and then pay by advertisers. For users, we only charge the cost of the network traffic, but the context-aware application will Take part of the user's traffic, and in the case of not informing the user, and sometimes may be contrary to the user's wishes, then the user is willing to pay for their perception of this part of the sale is also to be observed.

\section{Conclusion}

The behavior analysis of the user in the mobile context involves the analysis of the user and the user experience design. The difficulty lies in how to understand the various situations and the complicated environment of the user's use of the product. Now the use of more practice is through real-time research to understand the user's situation, and the cost is too high is the biggest problem of this approach, while the sample size is generally small, if you want to obtain large sample data will consume a lot of Resources. Therefore, from the perspective of the application of mobile devices, based on the knowledge of context awareness theory, the integration of data mining knowledge, while using a variety of research algorithm, can make the user data collection becomes more smooth and comprehensive, and thus more So that the product design and business value of the upgrade, this is the purpose of this article to achieve research. 


\section{Acknowledgements}

Hainan Higher Education Research Project: Hainan Province vocational skills competition management system, No. Hnky2016ZD-15.

\section{References}

[1] Yao Ning. Study on the construction of personal learning space of digital library based on situational awareness [J].Journal of Library Science, 2016 (10)

[2] Liu Hong. Study on personalized information recommendation service of digital library in colleges and universities based on situational awareness [J]. Library Journal, 2016 (08)

[3] Zhou Lingyuan, Wu Tingting, Jia Weiqiang. Application of Mobile Situational Perception Service in Museum - Taking Suzhou Museum APP as an Example [J]. Journal of Nanchang Hangkong University, 2016 (02)

[4] Jin Xiaopu, Bi Xin. Effects of user experience on mobile library service quality influencing factors [J].Information Theory and Practice, 2016 (06)

[5] Bi Qiang, Liu Jian. Digital literature resources content service recommendation method research [J]. Modern Library and Information Technology, 2015 (12)

[6] Zeng Ziming, Jin Peng. Intelligent Library personalized recommendation service system and model research [J]. Library Journal, 2015 (12) 